\title{
Projekt MARS - Statistiken der ambulanten Gesundheitsversorgung
}

\author{
Das Projekt MARS (Modules Ambulatoires des Relevés sur la Santé) des Bundes- \\ amtes für Statistik (BFS) verfolgt das Ziel, statistische Datengrundlagen zur Gesund- \\ heitsversorgung zur Verfügung zu stellen. Dadurch sollen die Öffentlichkeit, die Me- \\ dien, die Verbände und die Politik, durch datenbasierten Fakten unterstützt werden. \\ Gleichzeitig wird der Gesetzesauftrag des Krankenversicherungsgesetzes (Art. 22a) \\ umgesetzt.
}

Jacques Huguenin

Leiter Projekt MARS, Bundesamt für Statistik, Neuenburg
Korrespondenz:

Dr. Jacques Huguenin Bundesamt für Statistik BFS Sektion Gesundheit GES Espace de l'Europe 10 CH-2010 Neuchâtel Tel. 0584636614 Fax 0584636107 bfs.admin.ch
Ist die medizinische Versorgung angemessen und ausreichend für die Struktur der Bevölkerung? Wie sieht die Landschaft der Leistungserbringer in der Gesundheitsversorgung aus und wie entwickelt sie sich? Wie steht das Leistungsangebot im Verhältnis zur Versorgungsnachfrage? Wie entwickelt sich die Inanspruchnahme von bestimmten Leistungen? Werden stationäre Behandlungen durch ambulante ersetzt? Was sind die Auswirkungen für das Gesundheitssystem und für die Patientinnen und Patienten?

Um solche und weitere Fragen zu beantworten ist es unumgänglich, eine systematische und konsistente Datenbasis zur Verfügung zu haben. Das Bundesamt für Statistik verfolgt das Ziel, die Datengrundlage in der Gesundheitsversorgung bereitzustellen, um statistische Aussagen zu ermöglichen. Die zur Verfügung gestellte statistische Datenbasis dient der Öffentlichkeit (Medien, Verbänden oder Vertretern der Politik), um mit anerkannten, fundierten Fakten arbeiten zu können. Damit wird auch die öffentliche Debatte um die Gestaltung der Gesundheitspolitik gestützt. Wie kann beispielsweise der Zugang zur medizinischen Grundversorgung in hoher Qualität gesichert werden? Welche Bedürfnisse von medizinischer Aus- und Weiterbildung entstehen, um die Gesundheitsversorgung nachhaltig zu gewährleisten? Zudem erhält die Versorgungsforschung eine breite Datensammlung in Ergänzung zu registerbasierten Daten oder zu klinischen Studien.

\section{Gesundheitsversorgungsstatistik}

Das Bundesamt für Statistik erhebt bereits seit den 90er-Jahren Daten von Leistungserbringern im stationären Versorgungssektor, hauptsächlich bei Spitälern und Pflegeheimen. Diese beinhalten Angaben über die Betriebe, ihre Infrastruktur, das Personal, die Finanzen, sowie detaillierte Informationen über Behandlung und Morbidität der Patienten. Mit dem Projekt MARS werden die bestehenden Datensamm- lungen auf den ambulanten Versorgungssektor erweitert, um eine vollständige Gesundheitsversorgungsstatistik anbieten zu können. Das Projekt MARS ist in Teilprojekte gegliedert, die nach Leistungserbringer und nach Datentyp definiert sind. Diese Teilprojekte werden modular umgesetzt. In der ersten Priorität steht die Vervollständigung der Strukturund Patientendaten von Spitälern, gefolgt von Strukturdaten der Arztpraxen. Als Leistungserbringer sind die juristischen Personen, wie die Arztpraxis als Unternehmen, gemeint. Die Angaben zu den Ärztinnen und Ärzten als natürliche Personen werden anonymisiert, wie auch alle Angaben zu Patientinnen und Patienten. Die Erhebung von Strukturdaten der Arztpraxen umfasst Angaben über Infrastruktur und Ausstattung, Aus- und Weiterbildungsangebot, Umfang der Leistungen, Aus- und Weiterbildung des Personals sowie finanzielle Kennzahlen zu Aufwand und Ertrag. Diese Erhebung beinhaltet keine individuellen medizinischen Informationen über Patientinnen und Patienten.

\section{Auftrag des BFS}

Das Bundesamt für Statistik hat gemäss Bundesstatistikgesetz den Auftrag statistische Grundlagen im öffentlichen Interesse anzubieten. Im Bereich der Gesundheitsversorgung hat das BFS den spezifischen statistischen Auftrag zur Erstellung von Grundlagen für die Beurteilung von Funktions- und Wirkungsweise des Krankenversicherungsgesetzes. Dies erfordert eine Erweiterung der bestehenden Bundeserhebungen, um Aufschluss über die Inanspruchnahme der ambulanten Medizin und deren Gründe (Diagnosen), das Leistungsgeschehen sowie die damit verbundenen Kosten zu geben. Gleichzeitig erhebt das BFS Daten der Leistungserbringer nach dem Krankenversicherungsgesetz, um den gesetzlich vorgesehenen Empfängern die für ihren aufsichtsrechtlichen Auftrag notwendigen Daten weiterzuleiten (siehe Kasten zu den Gesetzesgrundlagen). 


\section{eine Befragung \\ Arzt/Ärztin wird im}

Fragebogen anonymisiert

\section{streng getrennte Verarbeitung und Nutzung}
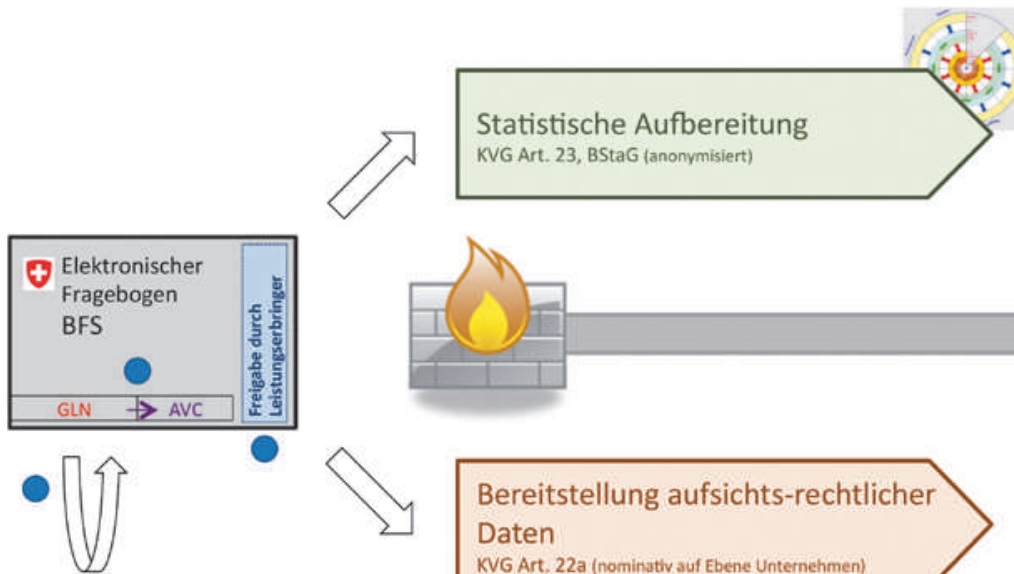

Bereitstellung aufsichts-rechtlicher

Daten

KVG Art. 22a (nominativ auf Ebene Unternehmen)
Statistische

Informationen Nutzung durch Offentlichkeit, OECD, Forschung, Bund Kantone,

Daten für administrative Zwecke Nutzung durch BAG, Kantone, u.a.

Webservice $\mathrm{FMH}$

Der Gesetzgeber hat dem BFS diesen doppelten Gesetzesauftrag erteilt, um Doppelspurigkeiten zu vermeiden, Synergien zu nutzen und dadurch den Aufwand der Datenlieferanten zu reduzieren. Das Bundesamt für Statistik setzt sich für diese Grundsätze ein wie auch für die strikte Einhaltung der Datenschutzbestimmungen.

\section{Projekt MARS}

Frühzeitig wurde im Projekt MARS eine Begleitgruppe mit Vertretern der verschiedenen Stakeholder gebildet. Dadurch sind die Partner informiert und in die Projektarbeiten integriert. Die Einführung einer jährlichen Vollerhebung kann nicht ohne zusätzlichen Aufwand bei den Datenlieferanten erfolgen. Dennoch ist es ein grosses Anliegen des Bundesamtes für Statistik, diesen Aufwand zu minimieren. Deshalb werden in der Regel Daten erhoben, die bereits in elektronischer Form bestehen und im Rahmen der Erhebung von Strukturdaten der Arztpraxen wird beispielsweise die Möglichkeit angeboten, durch einen Webservice der FMH auf bereits erfasste Datensammlungen zurückzugreifen. Um eine reibungslose Einführung zu sichern, werden solche Instrumente zur Erfassung und Erhebung von Daten in einer Pilotphase vertieft getestet. Ende 2015 soll mit der ersten produktiven Erhebung gestartet werden. Die Elemente der Erhebung erscheinen in der abgebildeten schematischen Darstellung des statistischen Prozesses: ein einziger Erhebungsvorgang für

\section{Statistische Gesetzesgrundlagen}

Bundesstatistikgesetz (BStatG, SR 431.01)

Der statistische Auftrag wird in BStatG Art. 3 (Aufgaben der Bundesstatistik) festgehalten. Die Grundsätze der öffentlichen Statistik stehen im BStatG und in der Verordnung über die Durchführung von statistischen Erhebungen des Bundes (Statistikerhebungsverordnung, SR 431.012.1). Die einzelnen Erhebungen und Statistiken sind im Anhang der Statistikerhebungsverordnung aufgelistet. Im Bereich der Gesundheitsversorgung sind es die Nummern 58 bis 62. Dort sind u.a. Gegenstand, Befragte und Modalitäten der einzelnen Erhebungen präzisiert.

\section{Bundesgesetz über die Krankenversicherung} (Krankenversicherungsgesetz KVG, SR 832.10) In KVG Art. 23 wird der statistische Auftrag an das BFS erteilt, Datengrundlagen zur Beurteilung der Funktions- und Wirkungsweise des Gesetzes nach den Grundlagen des BStatG zu erarbeiten.

KVG Art. 23 Statistiken

${ }^{1}$ Das Bundesamt für Statistik erarbeitet die notwendigen statistischen Grundlagen zur Beurteilung von Funktions- und Wirkungsweise dieses Gesetzes. Es erhebt zu diesem Zweck bei den Versicherern, den Leistungserbringern und der Bevölkerung die notwendigen Daten.

${ }^{2}$ Die befragten natürlichen und juristischen Personen sind zur Auskunft verpflichtet. Die Informationen sind kostenlos zur Verfügung zu stellen.

${ }^{3}$ Das Bearbeiten von Daten zu statistischen Zwecken erfolgt nach dem Bundesstatistikgesetz vom 9. Oktober 1992 
mehrere Zwecke, getrennte Verarbeitung der Daten für die Verwendung gemäss der unterschiedlichen Gesetzesgrundlagen, vereinfachte Datenerfassung durch die mögliche Nutzung eines Webservices und Anonymisierung der personenidentifizierenden Informationen.

Letztlich soll erreicht werden, dass mit Hilfe der Daten von Ärztinnen und Ärzten eine fundierte Debatte aufgrund von datenbasierten Fakten geführt

\section{Administrative Gesetzesgrundlagen}

Bundesgesetz über die Krankenversicherung (Krankenversicherungsgesetz KVG, SR 832.10)

Der Gesetzesauftrag zur Erhebung von Daten der Leistungserbringer zu aufsichtsrechtlichen Zwecken ist in KVG Art. 22a festgehalten.

KVG Art. 22a Daten der Leistungserbringer

Die Leistungserbringer sind verpflichtet, den zuständigen Bundesbehörden die Daten bekannt zu geben, die benötigt werden, um die Anwendung der Bestimmungen dieses Gesetzes über die Wirtschaftlichkeit und Qualität der Leistungen zu überwachen. Namentlich sind folgende Angaben zu machen:

a. Art der Tätigkeit, Einrichtung und Ausstattung, Rechtsform;

b. Anzahl und Struktur der Beschäftigten und der Ausbildungsplätze;

c. Anzahl und Struktur der Patientinnen und Patienten in anonymisierter Form;

d. Art, Umfang und Kosten der erbrachten Leistungen;

e. Aufwand, Ertrag und finanzielles Betriebsergebnis;

f. medizinische Qualitätsindikatoren.

${ }^{2}$ Die befragten natürlichen und juristischen Personen sind zur Auskunft verpflichtet. Die Angaben sind kostenlos zur Verfügung zu stellen.

3 Die Angaben werden vom Bundesamt für Statistik erhoben. Es stellt die Angaben nach Absatz 1 zur Durchführung dieses Gesetzes dem Bundesamt für Gesundheit, dem Eidgenössischen werden kann. Das Bundesamt für Statistik ist überzeugt, dass alle Akteure, einschliesslich der Leistungserbringer, von dieser Transparenz profitieren werden, letztendlich auch die Patientinnen und Patienten.
Preisüberwacher, dem Bundesamt für Justiz, den Kantonen und Versicherern sowie den in Artikel 84a aufgeführten Organen je Leistungserbringer zur Verfügung. Die Daten werden veröffentlicht.

${ }^{4}$ Der Bundesrat erlässt nähere Vorschriften zur Erhebung, Bearbeitung, Weitergabe und Veröffentlichung der Daten unter Wahrung des Verhältnismässigkeitsprinzips.

Die Veröffentlichung von nach KVG Art. 22a erhobenen Daten durch das BAG ist auf Verordnungsstufe geregelt. Die Verordnung über die Krankenversicherung (KVV, SR 832.102) sieht nominative Angaben über die Leistungserbringer oder ihre Kategorien vor.

KVV Art. 31 Veröffentlichung der Daten der Leistungserbringer ${ }^{1}$ Das BAG veröffentlicht die Ergebnisse der vom Bundesamt für Statistik gestützt auf Artikel 22a des Gesetzes und durch das BAG nach Artikel 51 des Bundesgesetzes vom 26. Juni 2006 über die universitären Medizinalberufe erhobenen Daten so, dass namentlich folgende Angaben oder Kennzahlen der sozialen Krankenversicherung nach Leistungserbringer oder nach Kategorien von Leistungserbringern ersichtlich sind:

a. Leistungsangebot der Leistungserbringer;

b. Diplome und Weiterbildungstitel der Leistungserbringer;

c. medizinische Qualitätsindikatoren

d. Umfang und Art der erbrachten Leistungen;

e. Kostenentwicklung.

\section{Aktuelle Forumthemen \\ $\sin$}

Diskutieren Sie mit! Im Forum präsentieren wir regelmässig brisante Themen aus Politik, Ökonomie und Wissenschaft, die das Schweizer Gesundheitswesen betreffen. Bringen Sie Ihre Meinung ein oder kommentieren Sie die Äusserungen Ihrer Kolleginnen und Kollegen. Das Forum finden Sie unter:

www.saez.ch/forum/ 